\title{
Contactless Conductivity Detector in PDMS Microfluidic System
}

\author{
Karolina Blaszczyk, Michal Chudy, Zbigniew Brzozka, Artur Dybko \\ Department of Microbioanalytics, Faculty of Chemistry, Warsaw University of Technology \\ Noakowskiego 3, 00-664 Warsaw, Poland \\ e-mail:dybko@ch.pw.edu.pl
}

\begin{abstract}
We present a new contactless conductivity detector for microfluidic system. In traditional conductivity detectors, the electrodes are in direct contact with analyte. In the presented microsystem, the electrodes are made of microchannels filled with solution of $\mathrm{KCl}$ - the technological solution called as pseudoelectrodes. Microfluidic system was fabricated in poly(dimethylsiloxane) PDMS, during a photolithography process. During the tests, dimension, type of the filling electrolyte and lay-out of pseudoelectrodes' microchannels were evaluated. Analyte was pumped into microchannels using a syringe pump. Repeatable changes in the signal were observed.
\end{abstract}

Key words: contactless conductivity detector, pseudoelectrodes, PDMS

\section{Introduction}

During last years we are observing dynamic development of a new large branch of analytical chemistry - lab-on-a-chip, based on the microfluidic structures [1]. The goal of lab-on-achip is to realize whole analytical steps in one microstructures i.e. sampling, mixing, separation, reaction and detection [2]. Initially channels with small diameter were made of silicon, but microstructures can also be made of another materials: ceramics, polymers and glass. Microfluidic structures can be made using several techniques from powder blasting, laser ablation, photolithography to lift-off [1].

Almost every type of detection technique was adapted from traditional analytical chemistry to lab-on-a-chip microdevices [2]. Among many available detections, electrochemical detection has gained more importance using analytical microdevices. Contactless conductivity detectors are developed intensively what is associated with their applications in microcapillary electrophoresis ( $\mu \mathrm{CE}$ ) [3]. In traditional conductivity arrangement, the electrodes are in direct contact with the solution under the test and there are several problems which can affect the measurements e.g. bubbles of gas from electrochemical reaction, the electrodes' surface characteristic may change in time as well as it is very difficult to isolate the electrodes from the potential used for separation in $\mu \mathrm{CE}$. These problems are eliminated in contactless conductivity detector. In this type of detection, there is no direct contact between the analyte and the electrodes, and the electric field used for separation does not have an impact upon detection. The electrodes of contactless detector may be placed under a microchannel [4], around a microchannel [5] and in plane of a microchannel [6].

\section{Experimental}

The paper presents a microfluidic system with contactless conductivity detector. The microsystem was developed in an elastomeric material, poly(dimethylsiloxane) (PDMS) (Sylgard 184, Dow Corning). Photolithography technique was used during the preparation of 
the microdevice. The mold was prepared from a dry photoresist with a thickness of $50 \mu \mathrm{m}$. A thickness of the photoresist determines a height of the microchannels. The pseudoelectrodes have a width of $50 \mu \mathrm{m}$ wheras the detection channel $100 \mu \mathrm{m}$. Liquid PDMS was prepared by mixing prepolymer with curing agent using the weight ratio of 10:1. After mixing air bubbles were removed using a vacuum desiccator. The mold was covered with liquid PDMS and cured for $2 \mathrm{~h}$ at $70^{\circ} \mathrm{C}$. Inlets and outlets were made by drilling at the ends of microchannels. Two PDMS plates, one with microgrooves and one with a flat surface, were bonded with usage of oxygen plasma generator and in these way hermetic microchannels were obtained. Fig. 1 presents a photograph of the prepared microsystem.

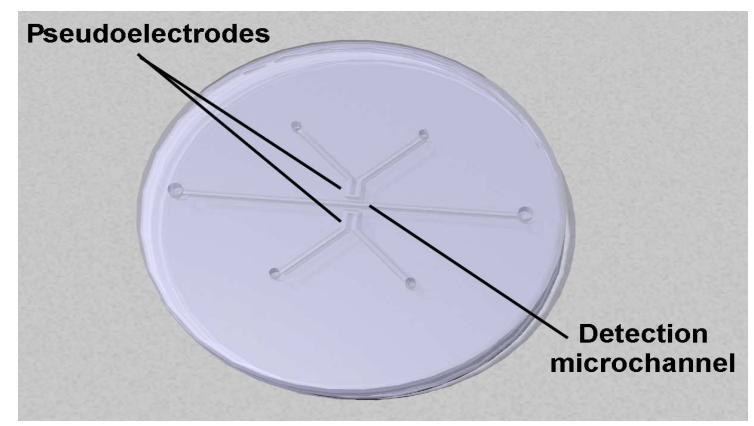

Fig. 1. A photograph of the developed microfluidic system with contactless conductivity detector

The developed microsystem consists of a detection channel and the electrodes which are placed on both sides of the detection channel. Typically in contactless detectors, the electrodes are fabricated from various metals. Since in this type of the detection there is no current flow between the electrodes, we decided to prepare them in form of microchannels filled with an electrolyte exhibiting high conductivity. This was the reason why we called them pseudoelectrodes (an expression liquid electrodes can also be used). Pseudoelectrodes' microchannels were obtained in the same way as measurement microchannels.

The distance between the detection microchannel and the pseudoelectrodes is equal to $75 \mu \mathrm{m}$. During tests we obtained results with four $1 \mathrm{M}$ electrolyte solutions into pseudoelectrodes' microchannels: $\mathrm{KCl}, \mathrm{CuSO}_{4}$, $\mathrm{NaOH}$ and $\mathrm{NaNO}_{3}$ (all analytical grades). 1M $\mathrm{KCl}$ solution was used for further research since the largest relative changes in the signal were obtained. The pseudoelectrodes were filled with $1 \mathrm{M}$ solution of $\mathrm{KCl}$ and wires were immersed into this solution in order to assure contacts between the pseudoelectrodes and measurement set-up (see fig. 2).

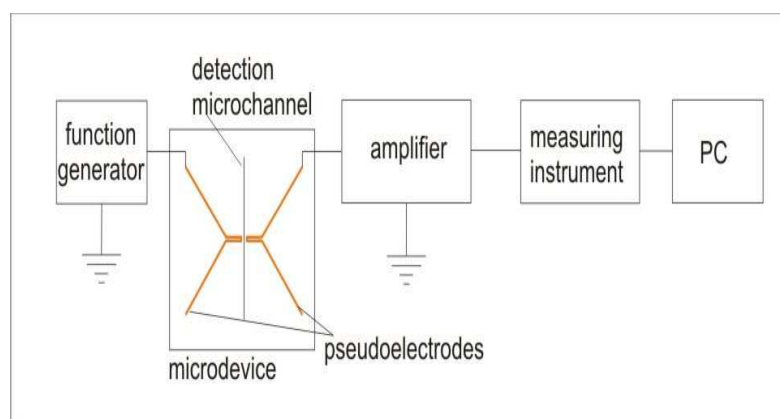

Fig. 2. A scheme of the measurement set-up.

During the tests, dimension, type of the filling electrolyte and lay-out of pseudoelectrodes' microchannels were evaluated. The fabricated microchannels were examined by optical, scanning electron and laser confocal microscopes (fig. 3). In order to test the usefulness of the developed microsystem, a model analyte (solution of $\mathrm{KCl}$ with various concentrations) was pumped through the detection microchannel by a syringe pump with rate of $50 \mu \mathrm{L} / \mathrm{min}$. Fig. 4 shows changes in the signal during pumping of various solutions.

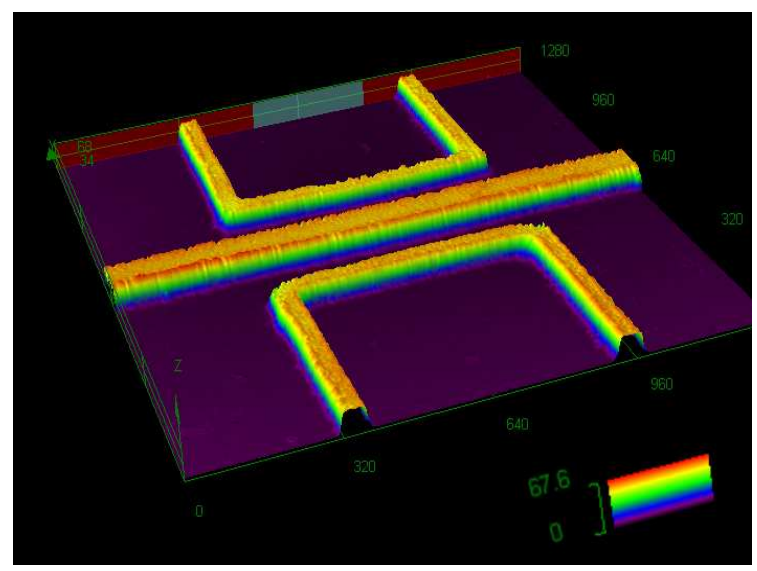

Fig. 3. A laser confocal examination of the detection zone.

Repeatable changes in the signal were observed. Having done the initial tests, we decided to cover the walls of the pseudoelectrodes with metal layer. A thin silver layer was fabricated by electroless deposition. However, the detector responses in both types of pseudoelectrodes were almost the same. Additionally, there was a problem with silver layer plaiting in elastomer since cracks in silver layer occurred very easily.

During the tests, evaporation of electrolyte from pseudoelectrodes' microchannels was observed. 


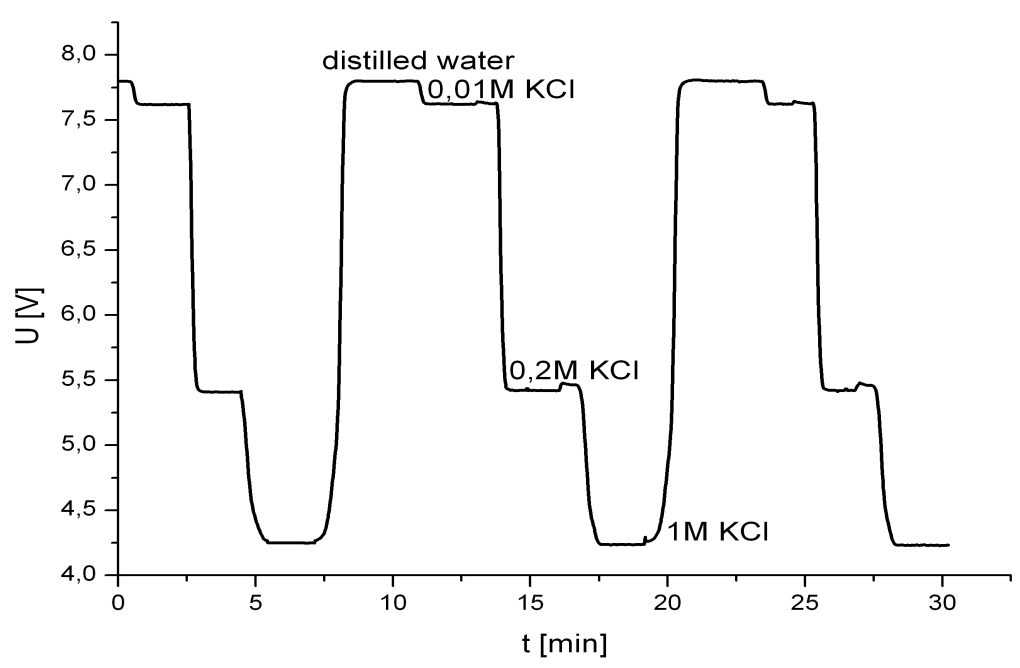

Fig. 4. A graph from analysis of $\mathrm{KCl}$ solution with usage of the developed detector.

There was small volume of electrolyte solution filling pseudoelectrodes' microchannel and evaporation during test had important influence on detector signal. Firstly, we covered inlets by parafilm and we observed a better stability of measurement. Therefore we decided to fill the pseudoelectrodes with agar-agar gel. We have used agar-agar dissolved in $1 \mathrm{M} \mathrm{KCl}$. We could observe bigger signal relative changes with agar-agar solution with $1 \mathrm{M} \mathrm{KCl}$ than electrolyte solution. The results are depicted in fig. 5 .

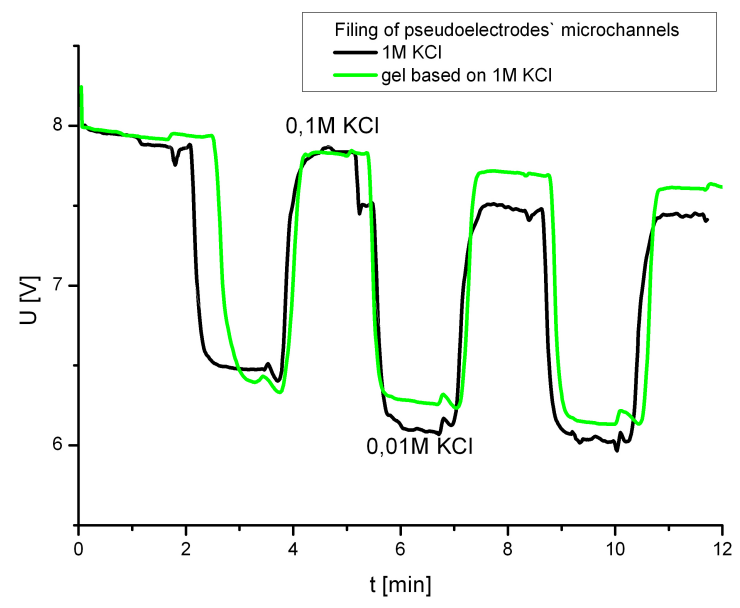

Fig. 5. Results of different filling of pseudoelectrodes microchannels.

\section{Conclusion}

It was proved that contactless conductivity detection with pseudoelectrodes is a good alternative to traditional conductivity detectors. Moreover, the presented technology is fast and inexpensive way for fabrication of conductivity microdetector. An additional advantage is a possibility of rapid adjustment of the shape and size of the pseudoelectrodes. Presented PDMS microsystem can be applied to various future applications.

\section{Acknowledgements}

This work was realized with a frame of project MNS-DIAG, which is financed by the European Union through the European Regional Development Fund and the Polish state budget in the framework of the Operational Programme Innovative Economy 2007-2013, contract No.UDA-POIG.01.03.01-00-014/08-01.

\section{References}

[1] D.R. Reyes, D. lossifidis, P.-A. Auroux, A. Manz, Anal. Chem. 74, 2623-2636 (2002), doi:

[2] P. Auroux, D. lossifidis, D.R. Reyes, A. Manz, Anal. Chem. 74, 2637-2652 (2002), doi: 10.1021/ac020239t

[3] J.A.F. da Silva, C.L. do Lago, Anal.Chem., 70, 4339-4343 (1998), doi: 10.1021/ac980185g

[4] R. M. Guijt, E. Baltussen, G. van der Steen, , Electrophoresis, 22, 2537-2541 (2001), doi: 10.1002/1522-2683(200107)22:12<2537::AIDELPS2537>3.0.CO;2-C

[5] P. Tůma, F. Opekar, I. Jelínek, Electroanalysis 13, 989-992 (2001), doi: 10.1002/15214109(200108)13:12<989::AIDELAN989>3.0.CO;2-C

[6] J. Lichtenberg, N.F. de Rooij, E. Verpoorte, Electrophoresis 23, 3769-3780 (2002) doi: 10.1002/1522-2683(200211)23:21<3769::AIDELPS3769>3.0.CO;2-E 